\title{
Emergency Transfemoral Amputation in a Severely Sick and Hemodynamically Unstable Patient through Eco- Guided Peripheral Nerve Block: Case Report
}

\author{
Luis Flavio Franca V Muniz ${ }^{1 *}$, Jessica Francisco Meireles ${ }^{2}$, Daissuke Kajita ${ }^{3}$ and Melissa \\ Ameloti G Avelino 4 \\ ${ }^{1}$ Department of Anesthesiology, Emergency Hospital of Goiânia, HUGO, Brazil \\ ${ }^{2}$ Department of Anesthesiology, Anesthesiology TEAM, Goiania, Brazil \\ ${ }^{3}$ Department of Vascular Surgery, Emergency Hospital of Goiania - HUGO, Brazil \\ ${ }^{4}$ Department of Otorhinolaryngology, PPGCS, HC/UFG, Brazil
}

\begin{abstract}
Critically ill and/or anticoagulated patients remain a great challenge for anesthesiologists regarding the anesthetic procedure. Its perioperative management should focus on organ preservation and avoid further damage. In this context, ultrasound-guided regional blocks are essential tools, as they avoid neuraxial invasion and deterioration in borderline hemodynamics, conferred by spinal anesthesia and general anesthesia, respectively. In this report, we present a case of a patient with septic shock, anticoagulated, and in need of an emergency surgical approach in the right lower limb in which ultrasound-guided peripheral nerve block was essential for a favorable outcome for the patient.
\end{abstract}

\section{OPEN ACCESS}

*Correspondence:

Luis Flávio França V Muniz,

Department of Anesthesiology, Coordinator of the Medical Residency Program (MRP) of Anesthesiology at the Emergency Hospital of Goiânia,

HUGO, Preceptor of the MRP of Anesthesiology, HC/UFG, Brazil,

E-mail: luisffranca@hotmail.com

Received Date: 05 Jul 2021

Accepted Date: 27 Jul 2021

Published Date: 02 Aug 2021

Citation:

Muniz LFFV, Meireles JF, Kajita D, Avelino MAG. Emergency Transfemoral

Amputation in a Severely Sick and Hemodynamically Unstable Patient through Eco-Guided Peripheral Nerve Block: Case Report. Clin Surg. 2021;

6: 3270.

Copyright $\odot 2021$ Luis Flavio Franca

$\checkmark$ Muniz. This is an open access article distributed under the Creative Commons Attribution License, which permits unrestricted use, distribution,

and reproduction in any medium, provided the original work is properly
Case Report: Patient in septic shock in need of amputation of the right lower limb at the level of the thigh, with unstable hemodynamics and severe respiratory conditions, undergoing ultrasoundguided peripheral nerve block, of the right femoral, sciatic and lateral cutaneous nerves of the thigh, as a single anesthetic technique.

Conclusion: Regional anesthesia of the peripheral nerve guided by ultrasound as a unique anesthetic technique, performed by experienced professionals or under supervision, is effective and safe for lower limb surgical procedures. We suggest this approach, especially in hemodynamically borderline patients or seriously ill with or without anticoagulation.

Keywords: Regional anesthesia; SIRS; SEPSES; Ultrasound; Femoral nerve; Ischiatic 2 nerve; Postoperative pain; Analgesia; Neuro-axial

\section{Introduction}

Ultrasound-guided peripheral nerve blocks are safe and effective anesthetic and analgesic techniques for upper and lower limb surgical procedures [3]. For more complex procedures, the neuro-axis anesthesia, such as rachis and epidural anesthesia are still used more frequently $[4,5]$. However, these procedures may impair hemodynamic and ventilatory stability or side effects that can increase the patient's comorbidities $[4,7]$.

Even today, we look for anesthetic interventions that impact the patient's homeostasis less without compromising the quality of anesthesia. Despite having a considerable number of published studies on regional anesthesia guided as a single anesthetic procedure, there is still a lack of data to encourage us to opt for them in critically ill or healthy patients $[1,3,4]$.

General anesthesia can be catastrophic in septic patients and with fragile hemodynamic due to systemic vasodilation and myocardial depression caused by the drugs used [4]. As of the review date of this article, we still have not found a robust number of publications in the international literature that support the practice of the usage of peripheral nerve block as the only anesthetic technique in severely ill patients.

\section{Objective}

To describe a successful experience in using isolated regional blockade guided echo as an 
anesthetic technique for the surgical procedure of the lower limb in a critically ill patient.

\section{Case Representation}

Male patient, 83 years old and 90 kilos, with an indication for right lower limb amputation at the thigh level, due to right foot necrosis with compromised proximal leg with an evolution to necrotizing fasciitis, severe sepsis, and shock septic. Truck driver patient, diabetic using oral hypoglycemic agents, has chronic atrial fibrillation, hypertension, and COPD due to smoking a pack of cigarettes a day for 40 years. Uses Rivaroxaban $20 \mathrm{mg}$ /day, Losartan $10 \mathrm{mg}$ /day, Amiodarone $200 \mathrm{mg}$ /day, Metformin $750 \mathrm{mg} /$ day. On his physical examination: HR $130 \mathrm{bpm}$, A.A heart rate (atrial fibrillation), axillary temperature 38 degrees, sleepy, blood pressure $90 \times 50$ $\mathrm{mmHg}$, poor peripheral perfusion with increased capillary refill time $(>5 \mathrm{~s})$. After an interview with the family and the vascular surgery team, we chose to block the echo-guided peripheral nerves due to the borderline situation of hemodynamics caused by the installed condition of septic shock. A double lumen catheter was inserted in the right internal jugular vein with the aid of ultrasound, and the protocol of septic shock was initiated. Simultaneously, we performed the peripheral nerve block with the aid of ultrasound. The patient was initially positioned in left lateral decubitus, and the sciatic nerve was approached through the right sub gluteal route with an identification of the nerve between the gluteus maximus and quadratus muscles femoral, close to the ischial tuberosity. After the identification of the nerve, we performed the infiltration of $15 \mathrm{ml}$ of $0.5 \%$ ropivacaine. The curved probe was used (3-5 Mhz) and 22 G Quincke rack needle.

Then, he was placed again in horizontal dorsal decubitus and with the probe straight ( $7 \mathrm{Mhz}$ to $13 \mathrm{Mhz}$ ), we identified the femoral nerve adjacent to the right femoral artery, in the iliac fascia compartment. Around the nerve, $20 \mathrm{ml}$ of the exact solution of ropivacaine were administered. We moved towards the superior lateral side with the same probe until we found the meeting point between the fasciae of the muscles [4] sartorius and fascialata tensor, in the anterolateral position of the thigh. At this point, we administered another $5 \mathrm{ml}$ of the local anesthetic solution to block the action of the lateral cutaneous nerve of the thigh. Antisepsis of all needle insertion fields (Quicke 22 G) with alcoholic Chlorhexidine solution two \% was done.

After about $20 \mathrm{~min}$, we tested the sensitive points with a needle $(25 \mathrm{G} \times 9 \mathrm{G}$ ), all mapping regions of blocked nerves (anterior and posterior thigh, medial, anterior, and lateral suprapatellar). All tests were negative, including the one of the cutaneous territory of the posterior part of the thigh; there was doubt due to the possibility of failure in the posterior femoral cutaneous nerve and on the medial surface of the upper patellar thigh, as the cutaneous territory of the inferior branch of the obturator nerve was not blocked in this case.

There was no hemodynamic damage (in fact, there was hemodynamic rescue due to fluid intervention), a 02-type nasal catheter was installed with the flow of 3 liters $/ \mathrm{min}$ and administered 2 $\mathrm{mg}$ of diazepam IV. The procedure lasted $60 \mathrm{~min}$ and elapsed without hemodynamic changes.

\section{Result}

Our pain assessment was restricted to $24 \mathrm{~h}$ after the surgery due to the permanence in the ICU and follow-up difficulties. However, we identified good analgesia; it was not administered any pain rescue drug, with $\mathrm{EVN}$ between 2 to 3 (EVN, scale numeric visual of pain, ranging from 0 to 10 , where 0 means no pain and ten means unbearable pain) [9]. There was no need for hemodynamic support with vasopressor drugs or ventilatory support. There were some oscillations of cognitive function and wakefulness in this period.

\section{Discussion}

Removing the infectious focus by ending the septic circuit alone would improve the patient's prognosis, but we believed that the technique proposed in this scenario was positive. In a way, we understood that the maintenance of the hemodynamic and ventilatory parameters added to little pain stimulus in the postoperative period. It also helped in the better prognosis of this patient.

Ultrasound-guided regional anesthesia of peripheral nerves has gained notoriety and defenders worldwide, showing itself as a safe and effective technique $[1,3]$. Despite its effectiveness and safety, traditional anesthesia applied to the neuroaxis could potentially risk hemodynamic imbalance, especially in this hemodynamically unstable patient [5].

A femoral nerve block is now widely accepted and standardized as an analgesic and anesthetic technique for the lower limbs above the knee and the sciatic nerve block for below-knee procedures. The femoral nerve is responsible for sensory and motor innervation of most of the femur, hip, extensor musculature of the leg, and skin of the anteromedial portion of the thigh. Moreover, the nerve sciatic nerve is responsible for most of the sensory and motor innervation of the leg and feet $[1,3,6]$.

Mamiya et al. studied peripheral nerve blocks in three severely ill patients, ages eighty or over and found benefits, both in associated comorbidities and analgesia. Karm et al. showed advantages in using the peripheral nerve block technique in a highly severe patient with cardiac dysfunction and anticoagulation.

Ultrasound also proved to be a safe technique in anticoagulated patients, especially in more superficial approaches and in sites of compressible punctures, although there is still no formal consensus on this theme $[2,8]$. We must always weigh the risks and the benefits for the patient in cases of anticoagulation, as in the case reported, where exposure to other anesthesia, such as neuraxial, could bring a greater risk of severe complications as a subarachnoid hematoma. In this report, we chose the unique anesthetic technique due to the patient's state of clinical degradation, where both the spinal anesthesia and general anesthesia with tracheal intubation could precipitate hemodynamic deterioration of the patient and promote other damage.

In specific cases, as described above, where surgical anesthesia does not impact physiological and metabolic function, peripherals regional blocks would be a good alternative. In this article, we can confirm it, though there is a need for new cases to confirm our proposal. We found evidence that peripheral nerve blocks guided by ultrasound performed by experienced physicians or under supervision are safe and effective techniques for analgesic procedures and as isolated anesthesia, especially in critically ill patients.

\section{Acknowledgment}

We thank the patient's family who consented to the writing and submission of this manuscript. We also thank our Senior Researcher for her support and guidance. And finally, the institution (HC/UFG) for technical and material support. 


\section{References}

1. Helayel PE, Conceição DB, Félix C, Boos GL, Nascimento BS, Filho GR Ultrasound-guided ischiatic-femoral block for amputation stub revision. Case report. Rev Bras Anesthesiol. 2008;58:480-4.

2. Martins LE, Ferraro LH, Takeda A, Munechika M, Tardelli MA Ultrasound-guided peripheral nerve blocks in anticoagulated patients case series. Rev Bras Anesthesiol. 2017;67(1):100-6.

3. Marhofer P, Greher M, Kapral S. Ultrasound guidance in regional anesthesia. Br J Anaesth. 2005;94(1):7-17.

4. Johnson RL. LKopp S, Duncan CM, Jacob AK, Erwin PJ. Neuraxial vs. general anesthesia for total hip and total knee arthroplasty: A systematic review of comparative-effectiveness research. Br J Anaesth. 2016;116(2):163-76

5. Hiroaki A, Masahiko S, Hiroki M, Shotaro A, Reo I, Kiyohide F, et al. Comparing outcomes after peripheral nerve block versus general anesthesia for lower extremity amputation: A nationwide exploratory retrospective cohort study in Japan. Reg Anesth Pain Med. 2020;45(6):399-404.

6. Tran DQ, Salinas FV, Benzon HT, Neal JM. Lower extremity regional anesthesia: Essentials four current understanding. Reg Anesth Pain Med $2019 ; 000019$.
7. Pisansky Andrew JB, Brovman Ethan Y, Kuo C, Kaye Alan D, Urman Richard D. Perioperative outcomes after regional versus general anesthesia for above the knee amputations. Ann Vasc Surg. 2018;48:53-66.

8. Fonseca MN, Pontes Jordão PJ, Perez VM, Alves RR, Fonseca GG. SBA 2020: Update on regional anesthesia guideline in anticoagulant use. Braz J Anesthesiol. 2020;70(4):364-87.

9. Veiga, D, Luis C, Relative D, Fernandes V, Botelho M, Santos P, et al. Postoperative delirium in critically ill patients: risk factors and outcomes. Rev Bras Anestesiol. 2012;62(4):469-83

10. Karm MH, Lee S, Yoon SH, Lee S, Koh W. A case report: The use of ultrasound-guided peripheral nerve block during above-knee amputation in a severely cardiovascular compromised patient who required continuous anticoagulation. Medicine (Baltimore). 2018;97(9):e9374

11. Mamiya K, Aono J. [Peripheral nerve blocks for lower limb amputation in three critically ill patients]. Masui. 2007;56(6):699-701. 